\title{
Effect of dosage increments on blood phenytoin concentrations
}

\author{
F. BOCHNER, W. D. HOOPER, J. H. TYRER, AND M. J. EADIE \\ From the Medical Professorial Unit of Queensland University, \\ Royal Brisbane Hospital, Brisbane, Australia
}

SUMMARY Blood phenytoin (diphenylhydantoin) concentrations were measured after each dosage change in 12 epileptic patients who were given increasing oral doses of phenytoin. In each of these patients a dosage increment beyond the dosage that produced a blood phenytoin level of 6-9 $\mu \mathrm{g} / \mathrm{ml}$. caused a disproportionately great increase in the blood concentration of drug. This effect might be expected if the limit of the body's capacity to metabolize phenytoin were being reached. As oral dosages were increased in one patient, measurements of the rate of urinary excretion of phenytoin metabolite showed that the phase of rapid rise in blood phenytoin concentration coincided with a failure to increase the rate of phenytoin metabolite excretion. Awareness of the non-linear relation between oral dose and blood concentration of phenytoin in the individual patient, and realization that the phase of rapid rise in blood phenytoin concentration occurs through the 'therapeutic' range of $10-20 \mu \mathrm{g} / \mathrm{ml}$., is of importance to those who use blood phenytoin levels as a guide to the adequacy of anticonvulsant therapy.

Neurologists have become increasingly interested in the measurement of blood concentrations of anticonvulsant drugs, and a good deal of information is now available about the more commonly used anticonvulsants, phenytoin (diphenylhydantoin) and phenobarbitone. It is known that, for these drugs, there is a correlation between blood level and therapeutic and toxic effects (Kutt and McDowell, 1968). The neurologist's main interest in using measurements of blood anticonvulsant levels is to help provide more effective treatment for patients with epilepsy, but in utilizing such data neurologists may not always be fully aware of all the pharmacological implications in the situations they are attempting to manipulate. One rather important example of such a situation lies in the matter of the relation between blood concentration of phenytoin and phenytoin dosage in individual patients. While data are available relating oral dosage to blood phenytoin level in groups of treated patients (Buchthal and Svensmark, 1960), and while Kutt (1971) has quoted rather general figures correlating blood level with oral dosage in adult patients which suggest that the correlation between the two may not be linear, relatively little appears to have been written specifically considering the effect on blood phenytoin concentration of increasing oral dosage in the individual patient. We became aware of this lack of information after some misadventures leading to phenytoin overdosage when we attempted to extrapolate from the blood phenytoin concentration at a particular oral dosage in patients to what the phenytoin concentration might be expected to be in those patients at higher dosages. We here wish to report some studies in which blood phenytoin concentration is correlated with increasing phenytoin dosage in individual patients.

\section{METHODS}

Blood phenytoin concentration was measured either by the Wallace (1968) spectrophotometric assay, or more recently, by the MacGee (1970) gas chromatographic assay. Urinary 5-(p-hydroxyphenyl)-5phenylhydantoin (HPPH) was measured by a slight modification of the MacGee (1970) assay, after acid hydrolysis of the HPPH glucuronide in urine. In both of the gas chromatographic assays 5-phenyl-5- 
(p-tolyl)-hydantoin was used as an internal standard. The method used for hydrolysing the glucuronide in urine may convert some minor phenytoin metabolites to HPPH (Chang, Savory, and Glazko, 1970), but the interest in the present study was in phenytoin metabolism as a whole rather than in particular metabolites, and it would seem reasonable to regard the HPPH assay as measuring the main urinary excretion product of phenytoin metabolism, a product accounting for approximately $75 \%$ of the phenytoin intake.

In 12 epileptic patients blood phenytoin concentrations were measured while at least two different oral dosages of phenytoin were taken, sufficient time (at least four days) being allowed to pass for the blood phenytoin concentrations to stabilize after a dosage change before measurement was undertaken.

In a single patient oral phenytoin intake was slowly increased by $100 \mathrm{mg} /$ day at intervals of approximately five days until a dosage of $500 \mathrm{mg} /$ day was taken, and then a further dosage increment of $30 \mathrm{mg} /$ day was made. In the earlier part of this particular study blood phenytoin concentration was measured daily and daily total urine HPPH output was measured. Later in the study, after the dose had reached $530 \mathrm{mg} / \mathrm{day}$, measurements were made at less frequent intervals. The study continued until toxic manifestations made a reduction in phenytoin dosage necessary.

\section{RESULTS}

In all 12 patients in whom blood phenytoin concentrations were measured at more than one oral phenytoin dosage it was found that, after attaining an oral dosage at which the blood phenytoin concentration reached a value of 6 to $9 \mu \mathrm{g} / \mathrm{ml}$., any further oral dosage increment (in general the increment was $100 \mathrm{mg} /$ day) tended to produce a more rapid rise in blood phenytoin concentration than had occurred with the previous dosage increment. Figure 1 shows data for five patients in whom blood phenytoin concentrations were measured at three or more dosage levels. The disproportionately rapid rise in blood phenytoin concentration with the final dosage increment was seen in all five subjects.

The patient studied in detail showed a relatively slow rate of increase in blood phenytoin concentration as oral dosage was increased in $100 \mathrm{mg}$ stages from 200 to $500 \mathrm{mg} / \mathrm{day}$. After two days at a dosage of $500 \mathrm{mg}$ phenytoin per day, blood concentration became steady for the next three days; a further $30 \mathrm{mg}$ dosage incre-

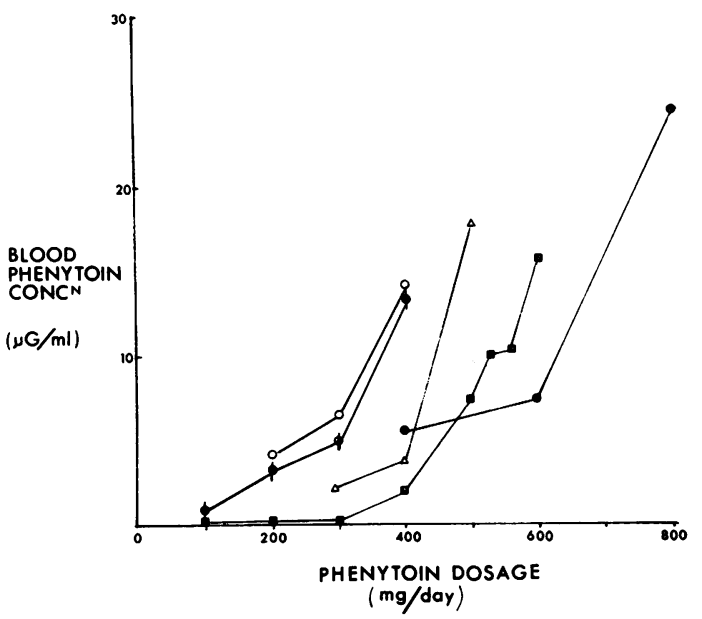

FIG. 1. Showing the increasingly rapid rates of rise of blood phenytoin concentrations in five patients as oral phenytoin dosages were increased.

ment then produced a very much more rapid rise in blood phenytoin concentration so thas $\omega$ from a blood level of $11.7 \mu \mathrm{g} / \mathrm{ml}$. at a dosage of 윽 $500 \mathrm{mg} /$ day the blood level rose to $26 \mu \mathrm{g} / \mathrm{ml}$. a市 a dosage of $530 \mathrm{mg} / \mathrm{day}$ - that is, a $6 \%$ dosage increment produced a more than $100 \%$ increase in blood phenytoin concentration. Coincident 3 with this rapid rise in blood phenytoin con centration, the rate of urinary excretion of the्य. metabolite HPPH, which had previously been increasing in proportion to dose, failed to show any further rise, suggesting that the rapid increase in blood level was related to failure to increase the rate of phenytoin metabolism (Fig. 2).

\section{DISCUSSION}

Previous studies (Buchthal and Svensmark, 1959) correlating blood phenytoin concentration with phenytoin dosage in groups of treated patients have shown considerable individual variation in response but an overall linear relation between dose and blood level. However, our findings, and those of Remmer, Hirschmann, and Greiner (1969) indicate that such a linear relation does not appear to apply in individual patients when their oral phenytoin dosages are progressively increased. In this circumstance the 


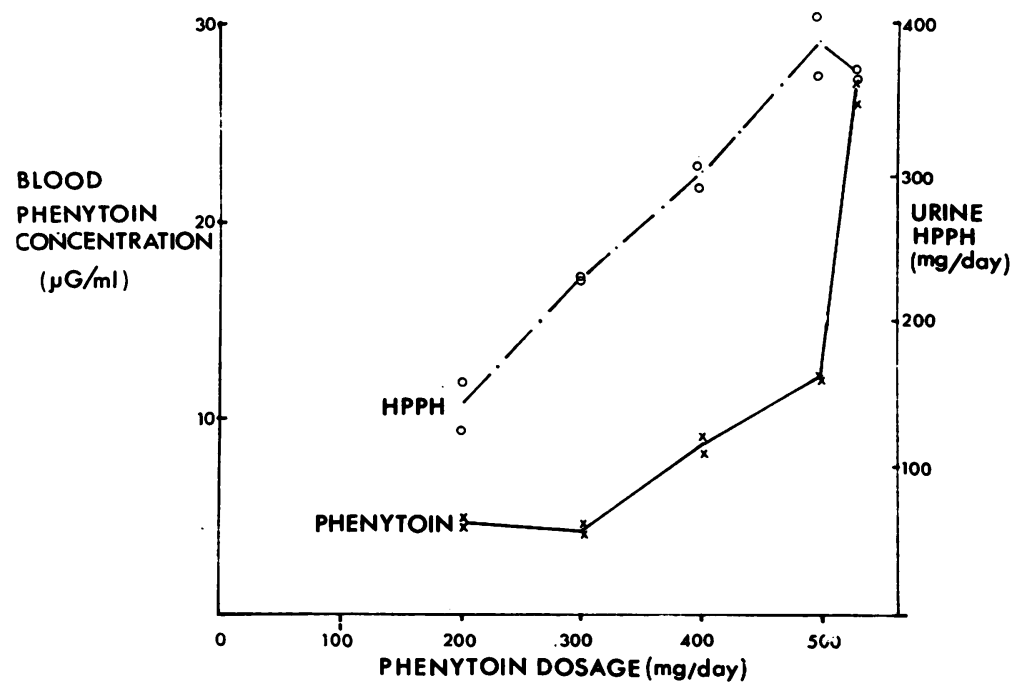

FIG. 2. Studies of blood phenytoin concentrations and urinary $\mathrm{HPPH}$ excretions in a patient given increasing oral phenytoin dosages. The rapid rise of blood phenytoin concentration at dosages over $500 \mathrm{mg}$ per day is associated with a failure to increase urine HPPH excretion. rate of rise in blood phenytoin level increases as the blood concentration of drug becomes higher. Such a finding might be anticipated, because it is known that the majority of the body's phenytoin intake is excreted in urine after enzymatic hydroxylation and glucuronide formation in the liver. An enzyme-catalysed reaction would be expected to have a maximum velocity, and once sufficient substrate (phenytoin) was present for this maximal velocity to be attained, any further phenytoin administered would tend to accumulate in the body and blood phenytoin concentration might be expected to rise with disproportionate rapidity compared with its previous rate of increase. The data of the present study bear out this theoretical expectation, in the patient investigated the rapid phase of blood phenytoin concentration increase being associated with a failure to increase the rate of excretion of phenytoin metabolite. This change from first order to zero order kinetics for phenytoin as blood concentration of the drug becomes higher may also explain Arnold and Gerber's (1970) data which showed that phenytoin half life $\left(\mathrm{T}_{\frac{1}{2}}\right)$ in blood increased as blood phenytoin level rose. In a sense, Arnold and Gerber (1970) were working in the reverse direction to the present study, and they did not measure phenytoin metabolism. However, from their data one might have suspected that blood phenytoin level would prove to rise out of proportion to dosage increase, and the present study has shown that this does happen.

The findings of the present investigation have some importance for the practising neurologist. At times blood phenytoin concentrations may rise slowly over several dosage increments and may not attain a sufficient level to prevent epilepsy, although the phenytoin dosage may be high by conventional standards. In this circumstance it may be thought that phenytoin is an ineffective anticonvulsant in a particular patient, yet further increment in phenytoin dosage may take the patient to the phase where blood phenytoin concentration will rise rapidly and a satisfactory therapeutic effect may occur with rather unexpected suddenness. Secondly, the rapid rise in blood phenytoin concentration with increasing dosage occurs through those phenytoin concentrations usually regarded as the 'therapeutic range' (10-20 $\mu \mathrm{g} / \mathrm{ml}$.). At times the usual phenytoin dosage increment of $100 \mathrm{mg} /$ day can take a patient from a sub-therapeutic phenytoin level to a toxic one, which can make it a matter of some difficulty to adjust blood phenytoin concentration to the desired therapeutic level. As a practical point, for those who are beginning to use blood phenytoin concentration as a guide to the adequacy of anticonvulsant therapy, it appears wise to make dosage incre- 
ments cautiously once blood phenytoin concentration exceeds $6-9 \mu \mathrm{g} / \mathrm{ml}$. if one does not wish to be confronted with unexpected phenytoin toxicity.

It would seem that knowledge of the behaviour of blood phenytoin concentration in individual patients in relation to oral dosage increments throws light on a number of effects of phenytoin therapy which, to the pharmacologically unsophisticated neurologist, may have appeared disproportionately severe in relation to the magnitude of the dosage changes he has prescribed.

\section{REFERENCES}

Arnold, K., and Gerber, N. (1970). The rate of decline of diphenylhydantoin in human plasma. Clinical Pharmacology and Therapeutics, 11, 121-134.
Buchthal, F., and Svensmark, O. (1960). Aspects of theō pharmacology of phenytoin $\left(\right.$ Dilantin $\left.^{R}\right)$ and phenobarbital relevant to their dosage in the treatment of(D) epilepsy. Epilepsia, 1, 373-384.

Chang, T., Savory, A., and Glazko, A. J. (1970). A new metabolite of 5,5-diphenylhydantoin (Dilantin). Biochemical and Biophysical Research Communication, 38,,$\widehat{0}$ 444-449.

Kutt, H. (1971). Biochemical and genetic factors regulating Dilantin metabolism in man. Annals of the New York $\frac{\hat{T}}{2}$ Academy of Sciences, 179, 704-722.

Kutt, H., and McDowell, F. (1968). Management of epilepsy with diphenylhydantoin sodium. Dosage regulation forproblem patients. Journal of the American Medical $\vec{F}$ Association, 203, 969-972.

MacGee, J. (1970). Rapid determination of diphenylhydan-O toin in blood plasma by gas-liquid chromatography. 흘 Analytical Chemistry, 42, 421-422.

Remmer, H., Hirschmann, J., and Greiner, I. (1969). Die Bedeutung von Kumulation und Elimination für dieळ Dosierung von Phenytoin (Diphenylhydantoin). Deutsche medinische Wochenschrift, 94, 1265-1272.

Wallace, J. E. (1968). Microdetermination of diphenyl- $\overrightarrow{0}$ hydantoin in biological specimens by ultraviolet spectrophotometry. Analytical Chemistry, 4, 978-980. 